\title{
The Influence of Compressive and Tensile Axial Strain on the Critical Properties of $\mathrm{Nb}_{3} \mathrm{Sn}$ Conductors
}

\author{
Bennie ten Haken, Arno Godeke and Herman H. J. ten Kate. \\ Applied Superconductivity Centre, University of Twente, P.O. Box 217, 7500 AE Enschede, The Netherlands
}

\begin{abstract}
Various $\mathrm{Nb}_{3} \mathrm{Sn}$ conductors are investigated in an axial strain experiment. The superconducting samples are soldered to a substrate that is bent to generate a compressive or tensile axial strain. Especially in the compressive strain range the critical current reduction is smaller than predicted by the well-known scaling law. The influence of the transverse strain components is investigated in a tape conductor by changing the thermal strain. It is found that the axial strain experiments can be described by an upper-critical field that depends on the deviatoric component of the strain tensor only. Finally a comparison of the critical temperature and the critical current as a function of axial strain is made.
\end{abstract}

\section{INTRODUCTION}

The influence of strain on the critical properties of $\mathrm{Nb}_{3} \mathrm{Sn}$ is an important item for the design of high-field superconducting magnets. For instance in the coils of the ITER plasma fusion reactor the $\mathrm{Nb}_{3} \mathrm{Sn}$ filaments are strongly compressed. The maximum axial strain that occurs in the superconductor is approximately $-0.7 \%$ and $-0.25 \%$ for a stainless steel and Incoloy jacket respectively. The scaling law used to predict the critical current in this case is mainly based on axial pull experiments where the maximum compressive strain is limited by the thermal compression of the matrix materials, typically around $-0.4 \%$ [1].

The effect of compressive axial strains can be investigated experimentally by connecting a conductor to a bendable substrate. Such an investigation was made previously on $\mathrm{Nb}_{3} \mathrm{Sn}$ tape conductors and a significant deviation of the scaling of the critical current from the well-known "scaling law" was found for a strong axial compression [2]. A comparison of these results with measurements on multifilamentary conductors is presented here. Moreover the influence of other (non-axial) strain components is investigated in a tape conductor, by changing the thermal strain that is applied by the sample holder. Finally a comparison is made between the critical temperature $\left(T_{c}\right)$ and critical current at a certain magnetic field $\left(I_{c}(B)\right)$ as a function of the axial strain.

\section{SCALING OF $B_{C 2}$ IN TAPE AND WIRE CONDUCTORS}

Various multifilamentary wires are soldered onto the U-shaped bending spring that is depicted in figure 1, after being heat-treated on a separate holder. An axial strain is induced in the wire if the spring is bent by an external force that acts on the two legs. This strain device and more details on the experimental procedure are described in a separate

Manuscript received October 17, 1994

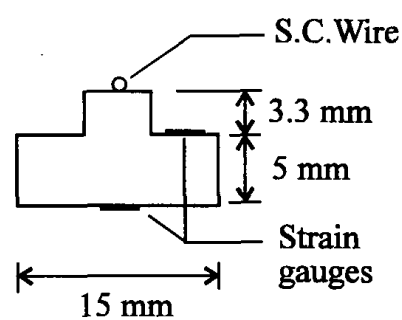

\section{Cross section of the top} of the U-spring for wires

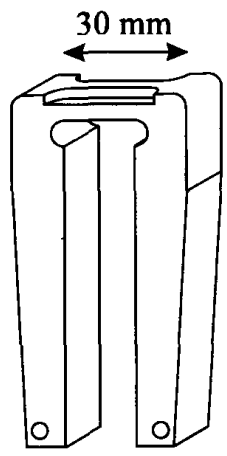

Fig. 1. The bending spring used for the strain experiments on wires.

study extensively [3]. The upper-critical fields of three different types of $\mathrm{Nb}_{3} \mathrm{Sn}$ multifilamentary wires are compared. Two different bronze route wires from Vacuum Schmeltze (VAC) and a wire produced according to the "Modified Jelly Role" process by Teledyne Wah Chang are investigated (TWCA). The VAC-NS wire has binary $\mathrm{Nb}_{3} \mathrm{Sn}$, the other two wires have ternary additions: VAC-HNST with $7.5 \% \mathrm{Ta}$ and TWCA with $1 \%$ Ti.

The upper-critical field $\left(B_{c 2^{*}}\right)$ is determined by means of a Kramer extrapolation of the critical current $\left(I_{c}\right)$ with constant coefficients:

$I_{c}^{0.5} B^{0.25} \propto B_{c 2^{*}}-B$

The $B_{c 2^{*}}$ as a function of the applied axial strain $\left(\varepsilon_{a}\right)$ is depicted in figure 2 . Note that a thermally contracted sample is defined here as: $\varepsilon_{a}=0$. It appears that the ternary additions cause a significant increase of the upper critical field over the entire investigated strain regime. The maximum in the $B_{c 2^{*}}$ of $\mathrm{Nb}_{3} \mathrm{Sn}$ tapes as previously presented lies between the binary $\mathrm{Nb}_{3} \mathrm{Sn}$ wire sample (VAC-NS) and the ternary $\mathrm{Nb}_{3} \mathrm{Sn}$ samples (TWCA and VAC-HNST).

The results obtained with VAC-NS wire are compared with Ekin's scaling law [1], where the $B_{c 2^{*}}\left(\varepsilon_{a}\right)$ dependence is fitted with a power of $u=1.7$. A typical example for such a power-law fit is represented by the dotted line in figure 2 . In this case the scaling constants (a) are taken as: 1000 for $\varepsilon_{a}-\varepsilon_{\max }<0$ and 1350 for $\varepsilon_{a}-\varepsilon_{\max }>0$. These values for $a$ are only slightly higher $(<10 \%)$ than those presented for 
other bronze route wires [1]. It can be concluded that the experimental results determined on this type of bending spring, coincide very well with the previously published results in the strain regime around the maximum $( \pm 0.4 \%)$.

For applied axial strains of -0.4 to $0.4 \%$ an almost linear $B_{c 2^{*}}\left(\varepsilon_{a}\right)$ dependence is found similar as in $\mathrm{Nb}_{3} \mathrm{Sn}$ tapes [2]. Such a linear dependence can be described with a power of $u=1$ in Ekin's scaling law. A transition from a power of approximately 1.7 to a power of 1 can be formulated with the following expression:

$B_{c 2^{*}}\left(\varepsilon_{a}\right)=B_{0, a}-C_{a} \sqrt{\left(\varepsilon_{a}+\delta\right)^{2}+\left(\varepsilon_{0, a}\right)^{2}}$,

where $\mathrm{d}$ is the axial thermal strain in the Nb3Sn filaments and an extra factor e0,a determines the $\mathrm{Bc} 2^{*}$ at ea $=-\mathrm{d}$. $\mathrm{A}$ least-square fit is made in the strain range above $-0.35 \%$ axial strain and the result is drawn in figure 2 .

The different constants determined for the $\mathrm{Bc} 2 *(\mathrm{ea})$ relation are summarised in table I. The proposed relation is a reasonable data fit, especially on the compressive side of the $\mathrm{Bc} 2 *$ maximum. The deviations that occur below $-0.35 \%$ strain are contributed to spatial inhomogeneities in the sample which leads to deviations in the Kramer extrapolation when $\mathrm{Bc} 2 *$ is close to the field where the Ic values are measured $(16 \mathrm{~T})$. In the strain range below $0.2 \%$ axial strain the description of equation 2 is a relatively good fit. Important for practical applications is that the $\mathrm{Bc} 2^{*}$, and thus the critical currents, in this strain range are significantly higher than predicted by the power law description.

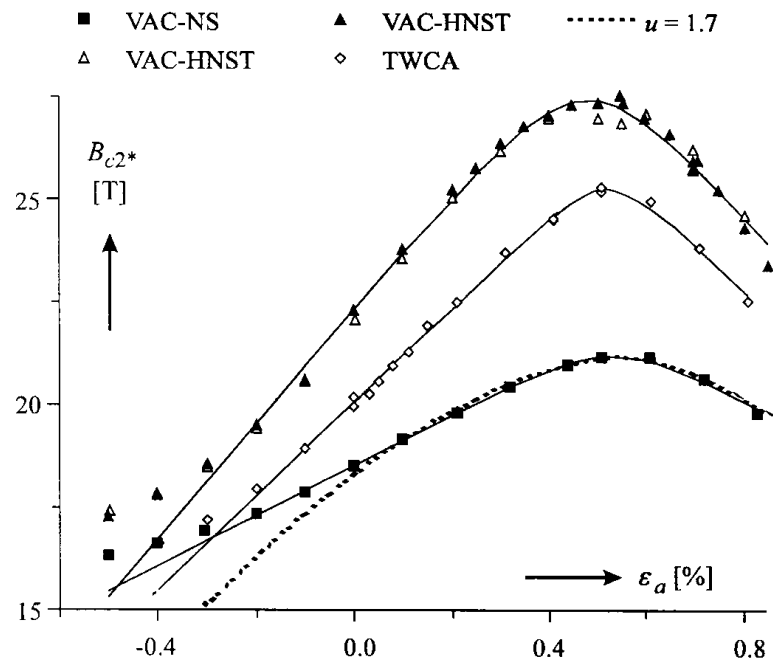

Fig. 2. The extrapolated upper-critical field determined in various multifilamentary wires. Extrapolation range: $B=10,12,14,16 \mathrm{~T}$. The $I_{c}$-criterion for the VAC samples is $10^{-3} \mathrm{~V} / \mathrm{m}$ and $10^{-4} \mathrm{~V} / \mathrm{m}$ for the TWCA wire. The strain state after cool-down is defined here as zero $\left(\varepsilon_{a}\right)$.
TABLE I

A SUMMARY OF THE PARAMETERS THAT DETERMINE $B_{c 2}\left(\varepsilon_{a}\right)$.

\begin{tabular}{lcccc}
\hline Sample Type & $B_{0, a}[\mathrm{~T}]$ & $C_{a}[\mathrm{~T}]$ & $\varepsilon_{0, a}[\%]$ & $\delta[\%]$ \\
\hline VAC-NS & 21.9 & 624 & 0.11 & -0.53 \\
VAC-HNST & 29.6 & 1440 & 0.15 & -0.49 \\
TWCA & 26.1 & 1150 & 0.08 & -0.52 \\
Tape (ref. [3]) & 22.7 & 890 & 0.10 & -0.52 \\
\hline
\end{tabular}

\section{Changing the Thermal Strain}

The influence of non-axial strain components can be investigated also in a bending spring-type experiment. If a thin tape is soldered onto a thick substrate (figure 4), then a thermal strain is induced in two directions inside the tape: $z$ (="axial") and $x$. In the $y$-direction, perpendicular to the tape, the strain is given by the Poisson's ratio $(v)$ of the $\mathrm{Nb}_{3} \mathrm{Sn}$. If the spring is bent there occurs an additional strain in the zdirection, but the strains in the other directions depend on the Poisson's ratio of the substrate $\left(v_{b}\right)$ :

$$
\left(\begin{array}{l}
\varepsilon_{x} \\
\varepsilon_{y} \\
\varepsilon_{z}
\end{array}\right)=\left(\begin{array}{c}
1 \\
-2 v /(1-v) \\
1
\end{array}\right) \delta+\left(\begin{array}{c}
-v_{b} \\
-v\left(1-v_{b}\right) /(1-v) \\
1
\end{array}\right) \varepsilon_{a}
$$

In this elastic model the thermal contraction of the sample holder will affect the thermal strain in the conductor.

The influence of the thermal contraction is investigated by comparing the $B_{c 2^{*}}$ measured on the brass bending spring $(\delta=-0.52 \%)$ with a stainless steel bending spring $(\delta=-0.32 \%)$. In this manner the uni-axial strain experiment is extended towards a two-component strain device.

The low conductivity of the substrate and the space required for the strain gauge between the voltage taps are in conflict with the limited sample size in the U-shaped bending spring. The $I_{c}$ in the strained section of the tape, can be much higher than the $I_{c}$ in the adjacent unstrained tape sections. A complication may arise with the transfer of the superconducting current from the superconducting layer to the normal matrix. This $I_{c}$ limit is more pronounced at lower magnetic fields, where the $I_{c}$ is large. Because of this field dependence, the $B_{c 2^{*}}$ extrapolation may give a higher value.

To minimise the influence of the entrance length, the $B_{C 2 *}$ is determined in a different manner. A Kramer extrapolation is made from 12 to $16 \mathrm{~T}$, with only a thermal compression acting on the sample $\left(\varepsilon_{a}=0\right)$. The slope (= pinning constant) that is obtained in this strain state is used to determine $B_{c 2^{*}}$ based on the $I_{c}$ that is measured at $16 \mathrm{~T}$. This alternative $B_{c 2^{*}}$ determination minimises the disturbances due to the limited length of the strained zone. 


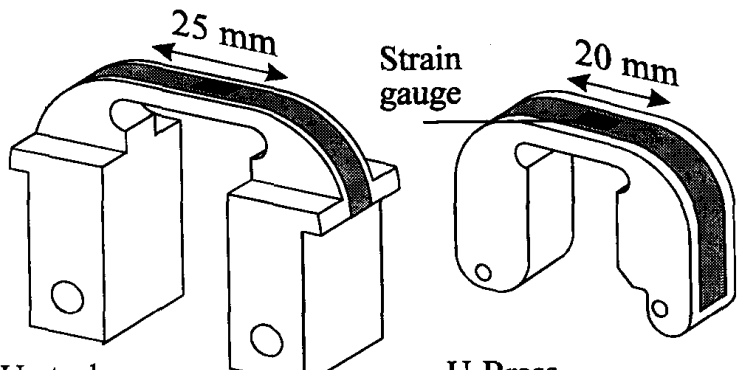

U-steel

U-Brass
Fig. 3. The bending springs used for the strain experiments on tapes.

The $B_{c 2 *}$ values measured in the tape samples on both different sample holders are shown in figure 5 The $B_{c 2 *}$ determined for the thermally compressed sample is approximately 3. $\mathrm{T}$ higher in the sample that is soldered on the stainless steel bending spring. This difference remains if the tape is strained to the $B_{c 2^{*}}$ maximum around $0.3 \%$ and $0.5 \%$ axial strain respectively. The large difference that occurs between the values of the two $B_{c 2^{*}}$ maxima can only be explained by nonaxial strain components $\varepsilon_{x}$ and $\varepsilon_{y}$ that are induced inside the $\mathrm{Nb}_{3} \mathrm{Sn}$ layer, because the axial strain $\left(\varepsilon_{z}\right)$ passes through zero in both cases.

The relation between the $B_{c 2 *}$ and the first two strain invariants is already investigated in transverse stress experiment. It appeared that the two parameters hydrostatic:

$\varepsilon_{\text {hyd }}=\varepsilon_{x}+\varepsilon_{y}+\varepsilon_{z}$,

and the "deviatoric" strain:

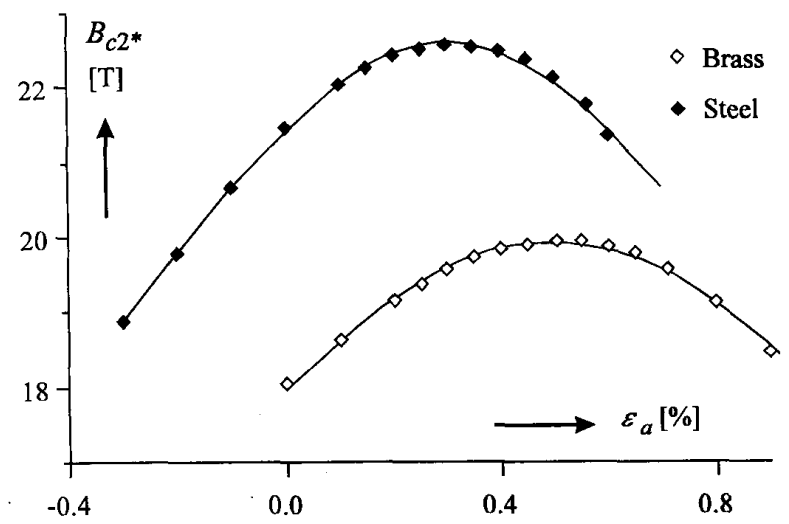

Fig. 4. The extrapolated upper-critical field as a function of the axial strain, measured on two different bending springs: U-Steel and U-Brass. $\varepsilon_{d e v}=\frac{2}{3} \sqrt{\left(\varepsilon_{x}-\varepsilon_{y}\right)^{2}+\left(\varepsilon_{y}-\varepsilon_{z}\right)^{2}+\left(\varepsilon_{z}-\varepsilon_{x}\right)^{2}}$,

could not describe the transverse pressure experiments properly [2]. The $B_{c 2^{*}}\left(\varepsilon_{a}\right)$ can be rewritten in terms of $B_{c 2^{*}}\left(\varepsilon_{d e v}\right)$ with the elastic tape model of equation 3. The Poisson's ratio of the $\mathrm{Nb}_{3} \mathrm{Sn}(v=0.40$, surveyed in [3]) in combination with a lower ratio for the sample holder $\left(v_{b}=0.25\right.$, measured on a brass spring at $4.2 \mathrm{~K}$ ), is applied here. For realistic values of the thermal strain on brass and steel of $-0.47 \%$ and $-0.29 \%$ respectively, a $B_{c 2}$ is found that depends linearly on the $\varepsilon_{d e v}$. This linear dependence, with a slope of $850 \mathrm{~T}$ is drawn in figure 6 . The lines through the measured $B_{c 2^{*}}\left(\varepsilon_{a}\right)$ points in figure 4 , are also calculated with this description. Regarding the tolerances in the mechanical model, the correlation between the measured curve and this linear description for $B_{c 2^{*}}\left(\varepsilon_{d e v}\right)$ is very good.

Based on the strain range that is investigated here one could extrapolate to a relatively high $B_{c 2^{*}}$ of approximately $27 \mathrm{~T}$ for a strain-free sample. If indeed the deviatoric strain component determines the $B_{c 2^{*}}$ then it is likely that there is a deviation from the proposed linear dependence for small deviatoric strains $\left(\varepsilon_{d e v}<0.4 \%\right)$. Moreover for a small deviatoric strain a slope $d B_{c 2^{*}} / d \varepsilon_{d e v}=0$, can be expected, in order to avoid singularities in the strain dependence of $B_{c 2^{*}}$.

An important consequence of the linear dependence of $B_{c 2^{*}}\left(\varepsilon_{d e v}\right)$ is that it predicts a rise in $B_{c 2^{*}}$ of a $\mathrm{Nb}+\mathrm{Nb}_{3} \mathrm{Sn}$ tape embedded between two $\mathrm{Cu}$ layers in a tape, if it is compressed in the transverse direction. Such a (reversible) rise in $I_{c}$ is not observed in a compressed tape [2], nor in any other conductor geometry. A satisfactory isotropic description that describes both the axial strain and the transverse stress experiments is not determined yet.

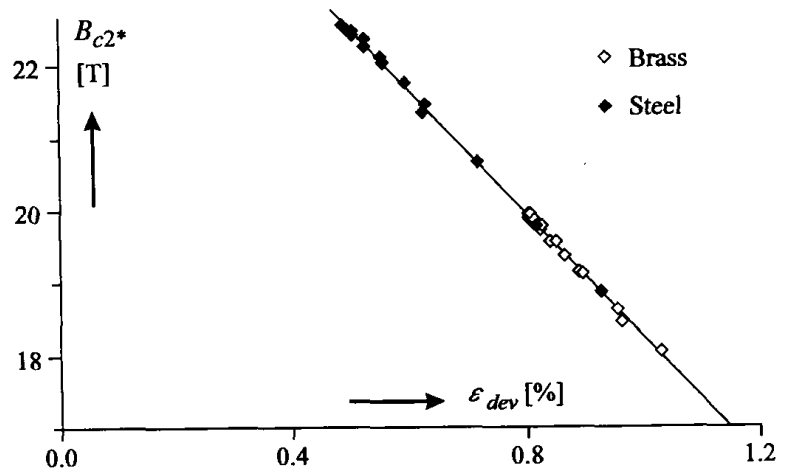

Fig. 5. The extrapolated $B_{c 2}$ as a function of the deviatoric strain calculated with the elastic model for the tape conductor. 


\section{COMPARISON OF $T_{C}, I_{C}$ AND $B_{C 2} *$ IN A NB ${ }_{3}$ SN TAPE}

It is usually assumed that the maxima in the $T_{c}\left(\varepsilon_{a}\right)$ and $I_{c}\left(\varepsilon_{a}\right)$ curves coincide at the same axial strain value, although in general the $T_{c}$ and $I_{c}$ strain dependencies are measured in different samples. However there is one reference where a difference in the position of the maximum in $T_{c}$ and $I_{c}$ was determined in a mono- and multifilamentary $\mathrm{Nb}_{3} \mathrm{Sn}$ conductor [4]. In order to clarify this the maxima of $T_{c}$ and $I_{c}(B)$ are investigated here in an axially strained $\mathrm{Nb}_{3} \mathrm{Sn}$ tape that also is used in previous strain experiments [2]. The $T_{c}$ is measured in zero field with an isolator cup covering the strain apparatus [3]. During the $I_{c}(B)$ determination this cup is removed by lifting it above the sample, in order to obtain a stable temperature of $4.2 \mathrm{~K}$. The $I_{c}(B)$ dependence is measured at 12,14 and $16 \mathrm{~T}$. The reproducibility of a $T_{c}$ determination, when repeated under exactly the same conditions, is rather good $(<5 \mathrm{mK})$.

The results are presented in figure 6 . The measured data points are compared with the proposed dependence for the $B_{c 2^{*}}\left(\varepsilon_{a}\right)$ that is also applied to the $T_{c}$ and $I_{c}$ data. A leastsquare fit results in a maximum in $B_{C 2}$ and $I_{c}$ that coincide at $\varepsilon_{a}=0.49 \%$ and for $T_{c}$ at $0.44 \%$. The difference in the position of the peak for $I_{c}$ and $T_{c}$ is visible but perhaps not significant because the missing $T_{c}$ points around $\varepsilon_{a}=0.4$. Nevertheless it is an indication that there could be such a difference. The difference that is observed here is in agreement with the experiments on bronze route $\mathrm{Nb}_{3} \mathrm{Sn}$ conductors as are described in the literature [4].

If it is assumed that there is a significant difference between the maxima in $T_{c}$ and $I_{c}$, then the discussion on the minimum-strain state starts to be very interesting. This could indicate that the different critical parameters $\left(T_{c}\right.$ and $\left.I_{c}\right)$ depend on different components of the strain tensor (e.g. the deviatoric and the axial strain). A physical mechanism

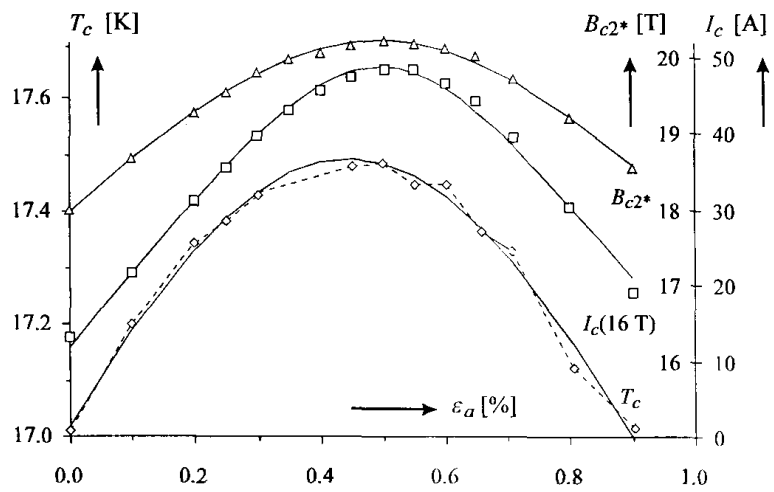

Fig. 6. A comparison of the $T_{c}, B_{c 2} *$ and $I_{C}$ versus axial strain measured in a $\mathrm{Nb}_{3} \mathrm{Sn}$ tape soldered on the U-Brass sample holder. that could predict such a behaviour is a strain- or stressdependent pinning force. An alternative mechanism to explain a difference between the $I_{c}$ and $T_{c}$ maxima is the different method that is used to determine both critical parameters. The $T_{c}$ is determined at the mid-value of the resistance transfer, corresponding to a sample that is for $50 \%$ in the resistive state. The $I_{c}$ is determined at the onset of the voltage-current transition, with only a small part of the sample in the normal state $\left(10^{-4} \mathrm{~V} / \mathrm{m}\right)$. If there are strain-induced inhomogeneities in the sample, then this can cause a difference in the strain dependence of both parameters.

\section{CONCLUSIONS}

1. The reduction of the $B_{c 2^{*}}$ in axially compressed $\mathrm{Nb}_{3} \mathrm{Sn}$ tape and wire conductors is less pronounced than predicted by the power law description for $B_{c 2^{*}}$ in Ekin's scaling law for $I_{c}(B)$ at high compressive strain. An alternative description for the axial strain dependence of $B_{c 2^{*}}$, with an almost linear dependence at high compressive strain, is proposed here.

2. Axial strain experiments on a $\mathrm{Nb}_{3} \mathrm{Sn}$ tape, that is deformed on two sample holders with a different thermal contraction, show a large influence of non-axial strain compo-

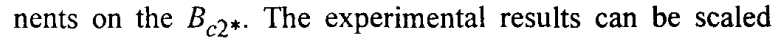
with a $B_{c 2^{*}}$ that depends linearly on the deviatoric strain component.

3. The deviatoric strain dependence for $B_{c 2^{*}}$ that is determined in the axial strain experiments is in contradiction with the $B_{c 2}$ reduction that is reported in transverse strain experiments.

4. A slight difference in the position of the $I_{c}$ and $B_{c 2^{*}}$ maximum and the $T_{c}$ maximum is found in a single axial strain experiment on a $\mathrm{Nb}_{3} \mathrm{Sn}$ tape. Further experiments are required in order to investigate this difference, especially the role of spatial inhomogeneities that influence the determination of the critical properties should be evaluated.

\section{REFERENCES}

[1] J.W. Ekin, "Strain scaling law for flux pinning in practical superconductors. Part 1 : Basic relationship and application to $\mathrm{Nb}_{3} \mathrm{Sn}$ conductors" Cryogenics, vol. 20, 1980, pp. 611 .

[2] B, ten Haken, A. Godeke and H.H.J. ten Kate, "The influence of various strain components on the critical parameters of layer shaped $\mathrm{Nb}_{3} \mathrm{Sn} ", A d v$. in Cryo. Eng. Vol 40 (Materials), 1993, pp 875-882.

[3] B. ten Haken, "Strain effects on the critical properties of high-field superconductors", Phd. Thesis, University of Twente, Enschede, The Netherlands, 1994.

[4] D.M. Kroeger et. al. "Evidence for microstructural effects under strain in bronze process $\mathrm{Nb}_{3} \mathrm{Sn}$ ", Filamentary A15 superconductors, $\mathrm{M}$. Suenaga and A.F. Clarck, p. 205, Plenum Press, USA (1980). 